Вячеслав А. Чепов, Иван И. Швецов-Шиловский, Сергей Б. Шмаков АЛГОРИТМ СООТНЕСЕНИЯ ФИЗИЧЕСКОЙ И ЛОГИЧЕСКОЙ АДРЕСАЦИЙ В МИКРОСХЕМАХ ПАМЯТИ С ПОМОЩЬЮ ИСТОЧНИКОВ ЛАЗЕРНОГО ИЗЛУЧЕНИЯ

\author{
Вячеслав А. Чепов ${ }^{1}$, Иван И. Швецов-Шиловский ${ }^{2}$, Сергей Б. Шмаков $^{3}$ \\ ${ }^{1,2,3}$ Начиональный исследовательский ядерный университет «МИФИ, \\ Каширское ш., 31, Москва, 115409, Россия \\ ${ }_{1,2,3}$ Акиионерное общество «Экспериментальное научно-производственное объединение \\ СПЕЦИАЛИЗИРОВАННЫЕ ЭЛЕКТРОННЫЕ СИСТЕМЫ», \\ Каширское ш., 31, Москва, 115409, Россия \\ 'e-mail:vache@spels.ru,http://orcid.org/0000-0002-5401-7102 \\ ${ }^{2}$ e-mail: iish@spels.ru,http://orcid.org/0000-0002-8161-9926 \\ 3 e-mail:sbshmak@spels.ru,http://orcid.org/0000-0002-4692-9355

\section{АЛГОРИТМ СООТНЕСЕНИЯ ФИЗИЧЕСКОЙ И ЛОГИЧЕСКОЙ АДРЕСАЦИЙ В МИКРОСХЕМАХ ПАМЯТИ С ПОМОЩЬЮ ИСТОЧНИКОВ ЛАЗЕРНОГО ИЗЛУЧЕНИЯ DOI: http://dx.doi.org/10.26583/bit.2020.3.04}

Аннотащия. В статье представлен разработанный алгоритм соотнесения физической и логической адресаций в микросхемах памяти с помощью источников лазерного излучения для определения характера сбоев при испытаниях на стойкость к ионизирующему излучению (ИИ). Предложен вариант аппаратно-программной реализации алгоритма, приведены ключевые программные инструменты. Алгоритм апробирован на источнике сфокусированного лазерного излучения с возможностью воздействия на отдельную ячейку памяти. Найдены закономерности расположения ячеек в блоке памяти, достаточные для построения полной карты соотнесения физической и логической адресации. Продемонстрированы основные результаты экспериментальной апробации алгоритма, которые подтверждают возможность разработки инструмента визуализации карты сбоев с точностью до одного бита информации. Показан пример применения данного инструмента для анализа стойкости микросхемы статического оперативного запоминающего устройства (СОЗУ) при воздействии импульсного ИИ.

Ключевые слова: ионизирующее излучение, физическая адресаџия памяти, логическая адресаџия памяти.

Для иитирования: ЧЕПОВ, Вячеслав А.; ШВЕЦОВ-ШИЛОВСКИЙ, Иван И.; ШМАКОВ, Сергей Б. АЛГОРИТМ СООТНЕСЕНИЯ ФИЗИЧЕСКОЙ И ЛОГИЧЕСКОЙ АДРЕСАЦИЙ В МИКРОСХЕМАХ ПАМЯТИ С ПОМОЩЬЮ ИСТОЧНИКОВ ЛАЗЕРНОГО ИЗЛУЧЕНИЯ. Безопасность информаиионных технологий, [S.l.], v. 27, n. 3, p. 43-53, 2020. ISSN 2074-7136. Доступно на: <https://bit.mephi.ru/index.php/bit/article/view/1291>. Дama docmyna: 02 sep. 2020.

DOI: http://dx.doi.org/10.26583/bit.2020.3.04.

Viacheslav A. Chepov ${ }^{1}$, Ivan I. Shvetsov-Shilovskiy ${ }^{2}$, Sergey B. Shmakov ${ }^{3}$

${ }^{1,2,3}$ National Nuclear Research University MEPHI (Moscow Engineering Physics Institute),

Kashirskoe sh., 31, Moscow, 115409, Russia

1,2,3 Joint Stock Company "Experimental Research and Production Association

SPECIAL ELECTRONIC SYSTEM",

Kashirskoe sh., 31, Moscow, 115409, Russia

'e-mail:vache@spels.ru,http://orcid.org/0000-0002-5401-7102

${ }^{2}$ e-mail: iish@spels.ru,http://orcid.org/0000-0002-8161-9926

3e-mail: sbshmak@spels.ru,http://orcid.org/0000-0002-4692-9355

\title{
Algorithm for matching physical and logical addressing in memory chips using laser sources DOI: http://dx.doi.org/10.26583/bit.2020.3.04
}

Abstract. The paper presents the developed algorithm for correlating the physical and logical addressing in memory chips using laser radiation sources to determine the nature of failures in radiation tests. A variant 
Вячеслав А. Чепов, Иван И. Швецов-Шиловский, Сергей Б. Шмаков АЛГОРИТМ СООТНЕСЕНИЯ ФИЗИЧЕСКОЙ И ЛОГИЧЕСКОЙ АДРЕСАЦИЙ В МИКРОСХЕМАХ ПАМЯТИ С ПОМОЩЬЮ ИСТОЧНИКОВ ЛАЗЕРНОГО ИЗЛУЧЕНИЯ

of the hardware-software implementation of the algorithm is proposed, key software tools are presented. The algorithm was tested using the focused laser facility with the ability to irradiate a separate memory cell. The patterns of location of the memory cells in single memory block were obtained, necessary to compose the full correlation between the physical and logical addressing. The main experimental results of the algorithm approbation are shown, which confirm the possibility of developing the tool for visualizing the map of failures with one-bit precision. The adaptation of the tool for analyzing the hardness of a static random-access memory (SRAM) IC under pulsed ionizing radiation is presented.

Keywords: ionizing radiation, physical memory addressing, logical memory addressing.

For citation: CHEPOV, Viacheslav A.; SHVETSOV-SHILOVSKIY, Ivan I.; SHMAKOV, Sergey B. Algorithm for matching physical and logical addressing in memory chips using laser sources. IT Security (Russia), [S.l.], v. 27, n. 3, p. 43-53, 2020. ISSN 2074-7136. Available at: <https://bit.mephi.ru/index.php/bit/article/view/1291>. Date accessed: 02 sep. 2020. DOI: http://dx.doi.org/10.26583/bit.2020.3.04.

\section{Введение}

В настоящее время существует проблема возникновения сбоев в микросхемах памяти при воздействии ионизирующего излучения $[1,2]$. Данная проблема существенно сказывается на сохранности информации: происходит потеря информации в ячейках памяти.

Соотнесение физической и логической адресаций в микросхемах памяти необходимо для построения пространственной карты распределения сбившихся ячеек, что дает возможность наглядно проанализировать характер сбоев, которые могут возникать при воздействии ионизирующего излучения. Визуализация сбившихся ячеек позволяет определять области на кристалле наименее стойкие к воздействию ИИ [3]. Результаты работы могут быть применимы в таких исследованиях как моделирование эффекта «просадки питания на внутренних шинах» (Rail-Span Collapse) [4, 5] в микросхемах, подверженных воздействию импульсного ИИ [6].

Знание взаимного расположения ячеек памяти между собой полезно, если стоит задача определения характера сбоев при воздействии отдельных заряженных частиц (ОЯч) [7]. В [8] представлена методика регистрации многократных сбоев (MC) в ячейках, относящихся к одному логическому блоку. Визуализация многократных физических сбоев позволит разработчику микросхем избегать многократные логические сбои путём выбора минимального расстояния между битами в слове (по одному адресу) $[9,10]$.

Одним из способов получения топологии является использование источника лазерного излучения. При заданных диаметре пятна и шаге сканирования лазерная установка имеет возможность регистрировать координаты ячеек памяти в месте воздействия лазерного излучения. После сканирования есть возможность построить карту, демонстрирующую соответствие координат и адресов сбившихся ячеек памяти. Однако для микросхем памяти с большой ёмкостью данный метод может занять большой промежуток времени (по расчётам для микросхемы СОЗУ ёмкостью 4 Мбит с проектными нормами 180 нм время сканирования заняло бы около 49 дней) [11].

В статье описан подход со значительным снижением временных затрат (до двух дней), при котором удается определить координаты каждой ячейки памяти, при воздействии только в часть накопителя без потери точности соотнесения физической и логической адресаций.

\section{1. Алгоритм соотнесения физической и логической адресаций}

Разработка алгоритма сопровождалась проведением эксперимента на сфокусированном источнике лазерного излучения. Данная установка облучает площадки микросхемы памяти при заданных диаметре пятна и шаге сканирования. При сканировании 
Вячеслав А. Чепов, Иван И. Швецов-Шиловский, Сергей Б. Шмаков АЛГОРИТМ СООТНЕСЕНИЯ ФИЗИЧЕСКОЙ И ЛОГИЧЕСКОЙ АДРЕСАЦИЙ В МИКРОСХЕМАХ ПАМЯТИ С ПОМОЩЬЮ ИСТОЧНИКОВ ЛАЗЕРНОГО ИЗЛУЧЕНИЯ

микросхемы регистрируются координаты в месте воздействия лазерного излучения. Таким образом, есть возможность построить карту, демонстрирующую соответствие координат и адресов сбившихся ячеек памяти.

У предлагаемого способа есть ряд ограничений:

- необходимость вскрытия микросхемы со стороны подложки для исключения влияния металлизации на равномерность облучения;

- необходимость наличия сбоя при выстреле в ячейку памяти.

Современные микросхемы памяти имеют достаточно большую ёмкость, поэтому облучение ячеек по всему размеру микросхемы представляется долгим процессом, вплоть до нескольких недель.

Принято решение производить воздействие лазерного излучения на микросхему следующим образом: выбирается размер пятна 3 микрометра, ячейки, подвергающиеся воздействию ИИ, расположены друг от друга на 100 микрометров вдоль осей абсцисс и ординат. В общем случае данные значения могут быть отличными от представленных. Диаметр пятна должен быть меньше расчётного размера ячейки памяти (n на рис. 1). Размер шага (m на рис. 1) выбирается при условии наличия нескольких сбоев вдоль осей абсцисс и ординат в пределах одного блока памяти. Остальные координаты ячеек с учётом симметрии блоков памяти достраиваются на карте сбоев, разработанной с помощью среды разработки NI LabVIEW 2018. Таким образом, нет необходимости сбивать все ячейки памяти в кристалле.

Сканирование проводится «змейкой»: после каждого воздействия ИИ на ячейку памяти лазер перемещается вдоль оси абсцисс с шагом m, пока не достигнет предела строки, после чего он с шагом $\mathrm{n}$ перемещается вниз. В каждой новой строке ячейки, подвергающиеся воздействию ИИ, смещаются вправо с шагом n. В итоге формируется последовательность, представляющая собой диагональные полосы сбившихся ячеек памяти по всему размеру кристалла. Данная последовательность позволяет сбить ячейки памяти вдоль осей абсцисс и ординат в пределах блока памяти, а значит является достаточным условием для анализа всего размера микросхемы памяти, что существенно сокращает время сканирования на сфокусированной лазерной установке.

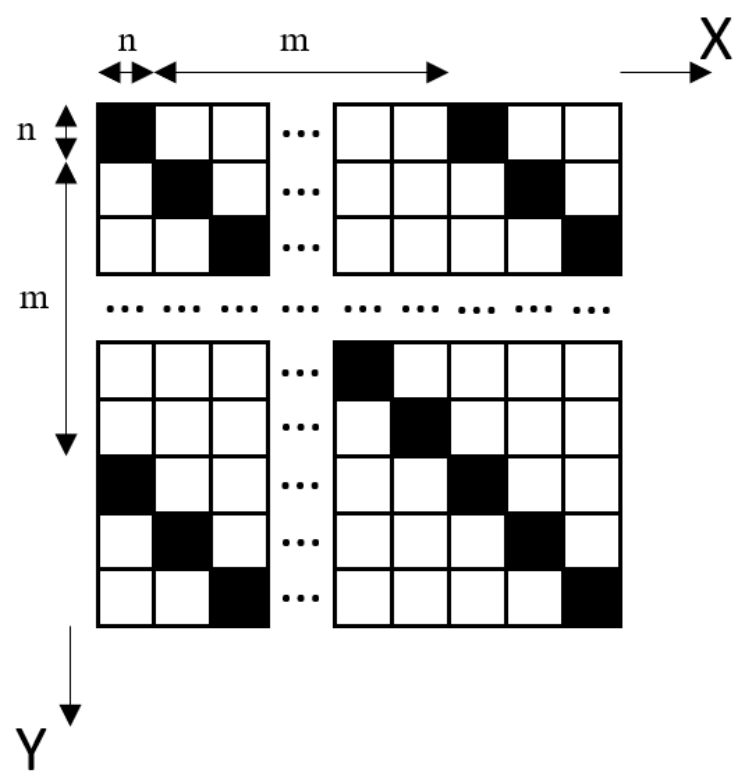

Рис. 1. Схематическое изображение заданной последовательности облучения ячеек памяти

(Fig. 1. Schematic representation of the given sequence of irradiation of memory cells) 
Вячеслав А. Чепов, Иван И. Швецов-Шиловский, Сергей Б. Шмаков

АЛГОРИТМ СООТНЕСЕНИЯ ФИЗИЧЕСКОЙ И ЛОГИЧЕСКОЙ АДРЕСАЦИЙ

В МИКРОСХЕМАХ ПАМЯТИ С ПОМОЩЬЮ ИСТОЧНИКОВ

ЛАЗЕРНОГО ИЗЛУЧЕНИЯ

Алгоритм соотнесения физической и логической адресаций:

1. Предварительный анализ

1.1. Анализ технической документации

1.1.1. Количество бит в строке/столбце

1.2. Визуальный анализ

1.2.1. Размер кристалла

1.2.2. Количество и объём банков памяти

1.2.3. Площадь одной ячейки

2. Выбор необходимой точности соотнесения. Выбор размеров пятна и шага сканирования при условии наличия хотя бы одного сбоя в пределах одного шага

3. Сканирование памяти на установке сфокусированного лазерного излучения. Получение карты сбоев (массив: координаты - адреса, по которым наблюдаются сбои)

4. Изменение разрядов адреса, определение, какие его разряды отвечают за:

4.1. Банк - при изменении адреса группа сбоев изменяет координату скачком

4.2. Строка - при фиксации разряда адреса и переборе его значений на карте наблюдается горизонтальная линия из сбоев, при изменении адреса эта линия смещается по вертикали

4.3. Столбец - при фиксации разряда адреса и переборе его значений на карте наблюдается вертикальная линия из сбоев, при изменении адреса эта линия смещается по горизонтали

5. Определение координат сбоев по адресам

5.1. Каждой комбинации адресов, отвечающих за столбцы, присвоить координату X

5.2. Каждой комбинации адресов, отвечающих за строки, присвоить координату Y

6. Дополнение адресной карты по выявленным закономерностям, если это необходимо

7. Если в строке/столбце/банке не было сбоя, то в результате анализа карты сбоев установить закономерности расположения строк/столбцов/банков и рассчитать координаты по адресам, где сбои не зафиксированы.

\section{2. Описание аппаратно-программного комплекса}

Блок-схема стенда для воздействия лазерного излучения на микросхему памяти приведена на рис. 2. Стенд содержит персональный компьютер, аппаратный комплекс фирмы National Instruments, сфокусированную лазерную установку ПИКО-3, лазерную установку РАДОН-8 [12, 13].

Аппаратный комплекс NI включает:

- PXI-4110 - 3-канальный программируемый источник питания

- PХI-7951R - ПЛИС (FPGA)

- PXI-6581 - 54-канальный несимметричный цифровой модуль адаптера вводавывода.

Задание необходимого уровня питания производится при помощи программируемого источника питания PXI-4110. С помощью ПЛИС PXI-7951R и цифрового адаптера ввода/вывода РXI-6581 проводится проверка сохранности информации. Обработка информации, полученной с аппаратного комплекса, и вывод результатов на экран монитора организуется посредством персонального компьютера. Воздействие лазерного излучения на микросхему памяти осуществляется с помощью сфокусированной лазерной установки ПИКО-3 или лазерной установки РАДОН-8. 
Вячеслав А. Чепов, Иван И. Швецов-Шиловский, Сергей Б. Шмаков АЛГОРИТМ СООТНЕСЕНИЯ ФИЗИЧЕСКОЙ И ЛОГИЧЕСКОЙ АДРЕСАЦИЙ В МИКРОСХЕМАХ ПАМЯТИ С ПОМОЩЬЮ ИСТОЧНИКОВ ЛАЗЕРНОГО ИЗЛУЧЕНИЯ

Разработка ПО проводилась с помощью среды разработки NI LabVIEW 2018. Блоксхема ПО приведена на рис. 3. Основной частью разработанного ПО является программа, отвечающая за управление всеми РХІ-модулями, входящими в состав стенда.

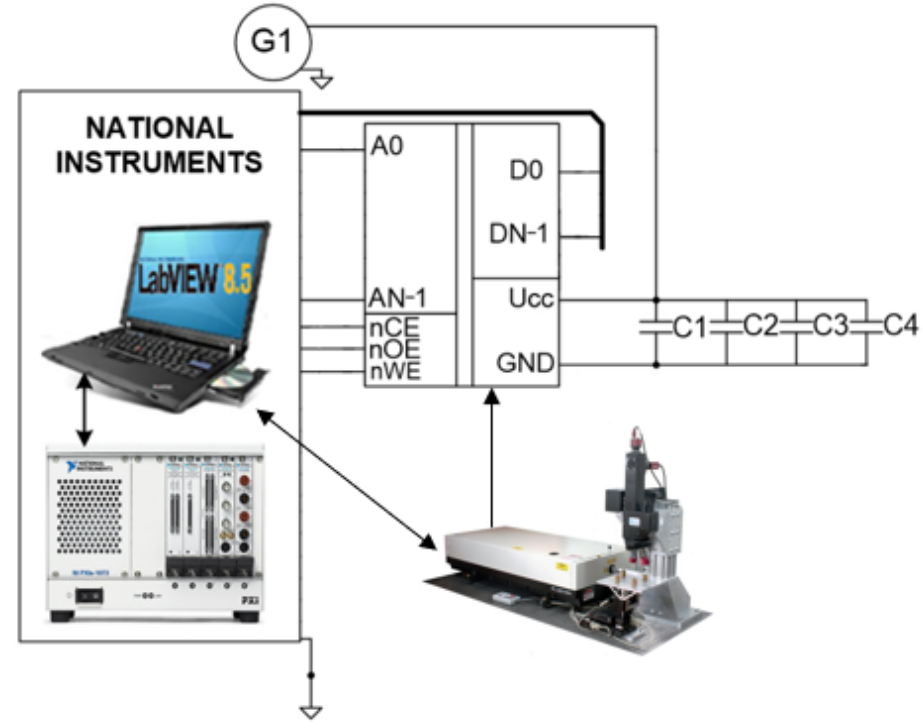

Рис. 2. Блок-схема стенда

(Fig. 2. Block diagram of the test setup)

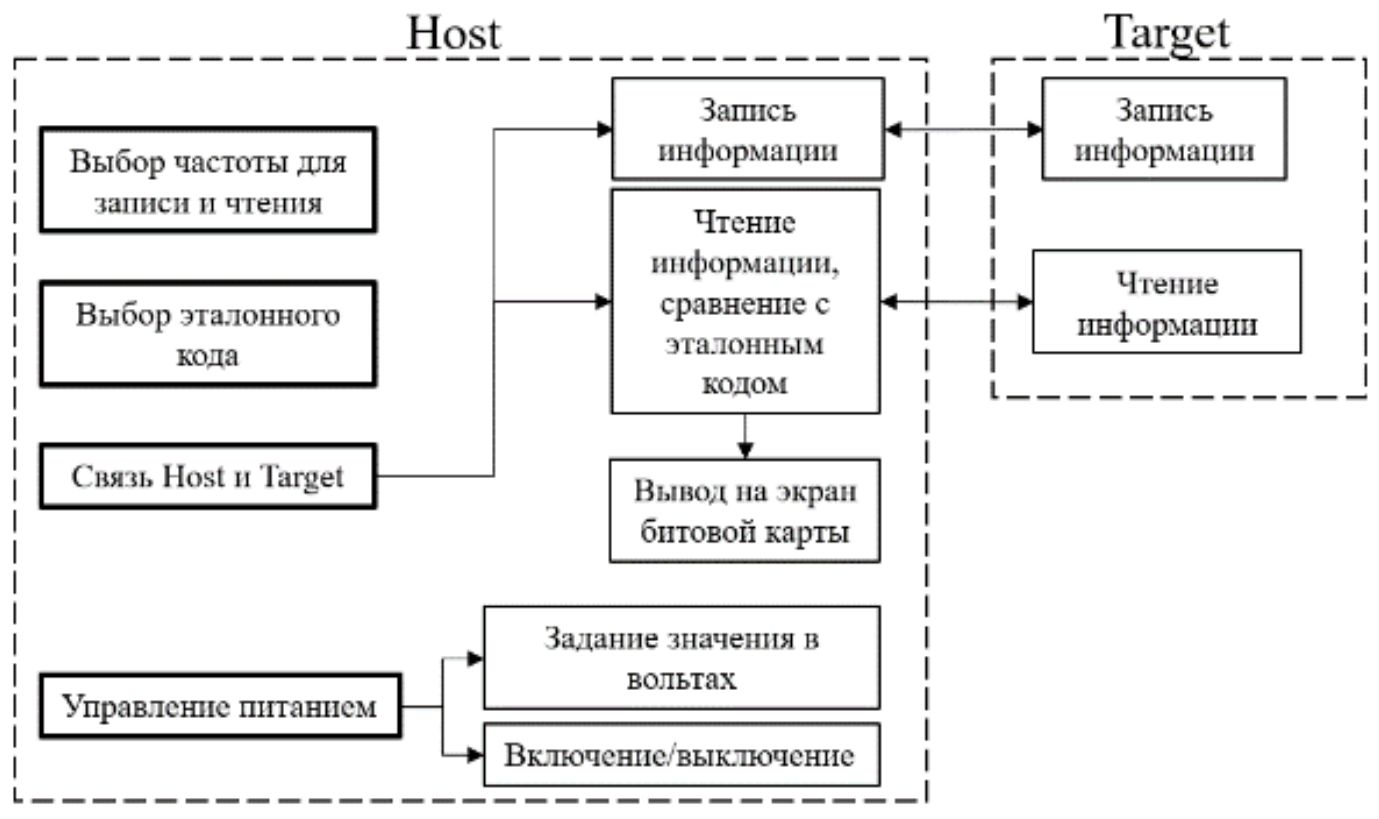

Рис. 3. Блок-схема ПО, иллюстрирующая ключевые функииональные блоки

(Fig. 3. Software block diagram illustrating key functional blocks)

\section{3. Программа формирования карты сбоев}

В данной программе предусмотрена возможность интерактивно взаимодействовать с каждым разрядом адреса памяти. Для этого добавлены управляющие элементы, число которых равно количеству разрядов адреса памяти (для исследуемой памяти с объёмом 
Вячеслав А. Чепов, Иван И. Швецов-Шиловский, Сергей Б. Шмаков АЛГОРИТМ СООТНЕСЕНИЯ ФИЗИЧЕСКОЙ И ЛОГИЧЕСКОЙ АДРЕСАЦИЙ В МИКРОСХЕМАХ ПАМЯТИ С ПОМОЩЬЮ ИСТОЧНИКОВ ЛАЗЕРНОГО ИЗЛУЧЕНИЯ

4 Мбит их число равно 22). Также в программу добавлена адресная строка, предоставляющая возможность вводить интересующий адрес в виде двоичного кода.

Переключение управляющих элементов в активное состояние интересующих разрядов адреса фиксирует данные разряды. В результате этого формируется пара масок. Фиксированные разряды адреса строго демонстрируют те значения, которые указаны в адресной строке. Оставшаяся часть разрядов принимает значения, соответствующие типу маски (для Mask1 - единицы, для Mask0 - нули).

Программа проводит проверку соответствия масок с каждым адресом памяти. При фиксировании управляющими элементами интересующих разрядов адреса на карте сбоев отобразятся только те координаты ячеек памяти, которые соответствуют указанным значениям разрядов в адресной строке. Множество координат ячеек памяти, разряды адреса которых не были зафиксированы управляющими элементами, отображаются на карте сбоев в полном объеме.

Иными словами, на карте сбоев отображаются координаты ячеек памяти, которые удовлетворяют условию:

(Маска единиц | Адрес = Маска единиц $) \&($ Маска нулей \& Адрес = Маска нулей $)$, где Маска единиц - адрес, все разряды которого равны единице кроме фиксированных;

Маска нулей - адрес, все разряды которого равны нулю кроме фиксированных;

Адрес - адрес облученной ячейки памяти, подающийся на сравнение с масками; |, \& - логические операторы.

\section{4. Программа вывода изображения}

Программа вывода изображения иллюстрирует аналитически заданную последовательность булевых констант, где константы со значением ИСТИНА показывают расположения сбившихся ячеек памяти. Разработка программы проводилась при помощи анализа карты сбоев, описанной выше.

Изображение формируется путём конвертирования из двумерного массива. Таким образом, основная идея задания необходимой аналитической последовательности состоит в том, чтобы при изменении значения определённых разрядов адреса выделить закономерности изменения расположения координат облученных ячеек памяти относительно оси абсцисс и ординат.

Анализ карты сбоев позволил разделить разряды адреса на группы: блоки памяти, строки, столбцы, биты данных. Данное разделение объясняется различием характера закономерности каждой группы. С учетом полученных в ходе анализа закономерностей значения адресов образуют массивы данных для каждой группы.

Элементы каждого образованного массива проходят проверку соответствия с адресом, у которого зафиксированы разряды, соответствующие определённой группе. Таким образом образуется пара масок (маска единиц и маска нулей). Маски сравниваются со значением адреса, пришедшего на вход программы согласно условию, описанному выше:

(Маска единиц | Адрес = Маска единиц $) \&($ Маска нулей \& Адрес = Маска нулей).

После завершения данных операций проводится поиск значения, которое удовлетворяет условиям для всех групп, которые соответствуют изменению координат относительно оси абсцисс, и выносится его индекс. Такая же процедура предусмотрена для групп, соответствующих изменению координат относительно оси ординат. В блок замены массива заносятся найденные индексы строк и столбцов. Таким образом, в двумерном массиве появляется булева константа ИСТИНА, иллюстрирующая расположение координаты сбившейся ячейки памяти. Результатом перебора всего 
Вячеслав А. Чепов, Иван И. Швецов-Шиловский, Сергей Б. Шмаков

АЛГОРИТМ СООТНЕСЕНИЯ ФИЗИЧЕСКОЙ И ЛОГИЧЕСКОЙ АДРЕСАЦИЙ

В МИКРОСХЕМАХ ПАМЯТИ С ПОМОЩЬЮ ИСТОЧНИКОВ

ЛАЗЕРНОГО ИЗЛУЧЕНИЯ

множества адресов, пришедших на вход программы, является двумерный массив булевых констант, значения ИСТИНА которого демонстрируют координаты сбившихся ячеек памяти.

\section{5. Основные результаты апробации алгоритма}

С помощью лазерной установки ПИКО-3 проведено облучение кристалла по заданным размерам пятна и шага сканирования. Результаты облучения занесены в логфайл, в каждом абзаце которого располагаются адреса облученных частей кристалла и координаты точек, в которых эти части были получены. Кроме того, в программном обеспечении на лазерной установке проиллюстрировано изображение координат сбившихся ячеек памяти (рис. 4).

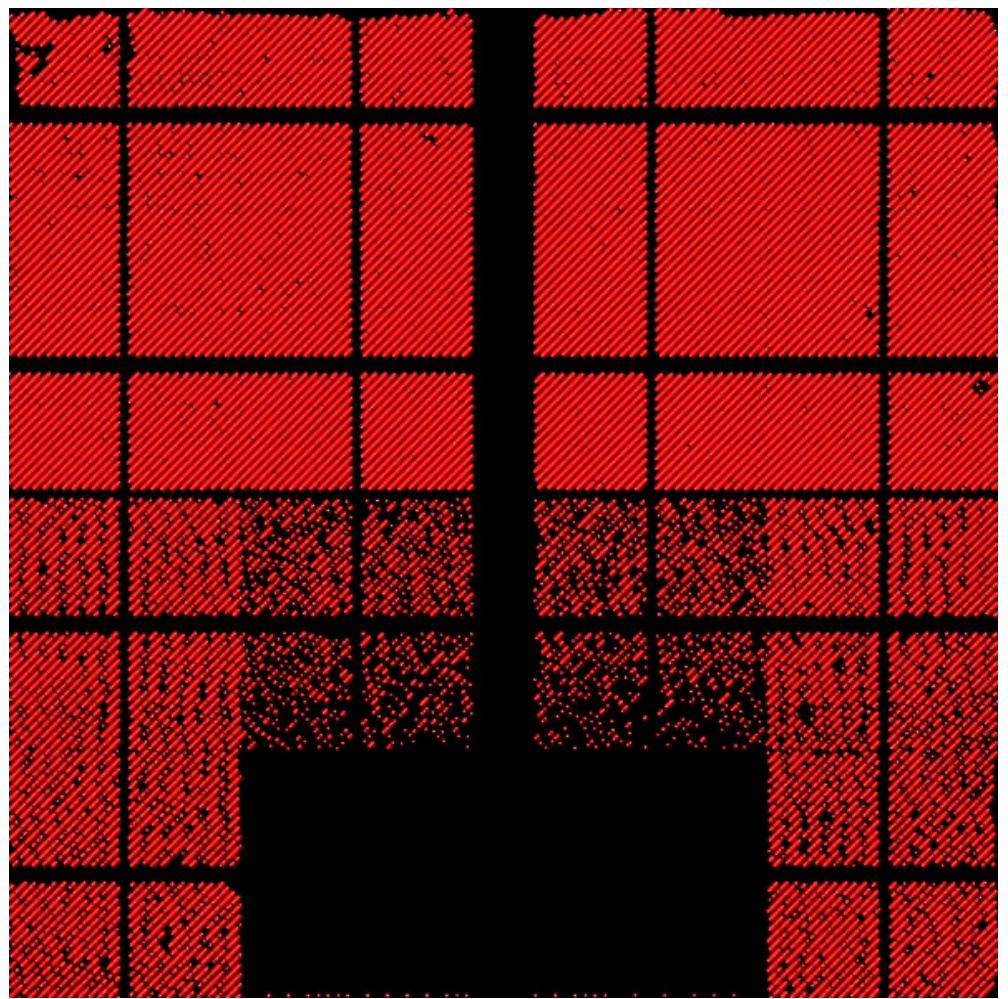

Рис. 4. Изображение координат сбившихся ячеек памяти, полученное в ПО на сфокусированной лазерной установке

(Fig. 4. Image of the coordinates of upsets in memory cells obtained by software on a focused laser system)

На рис. 4 видно, что в нижней области нет отображения некоторых координат (чёрный прямоугольник). Связано это с нехваткой энергии при лазерном облучении данного участка памяти.

Занесенные в лог-файл адреса облученных частей кристалла и соответствующие им координаты были поданы на вход программы формирования карты сбоев. Полученные данные дали возможность построить карту сбоев, представленную на рис. 5. Карта в точности иллюстрирует изображение, полученное на сфокусированной лазерной установке. 
Вячеслав А. Чепов, Иван И. Швецов-Шиловский, Сергей Б. Шмаков

\section{प \\ 0000000000000000000100}
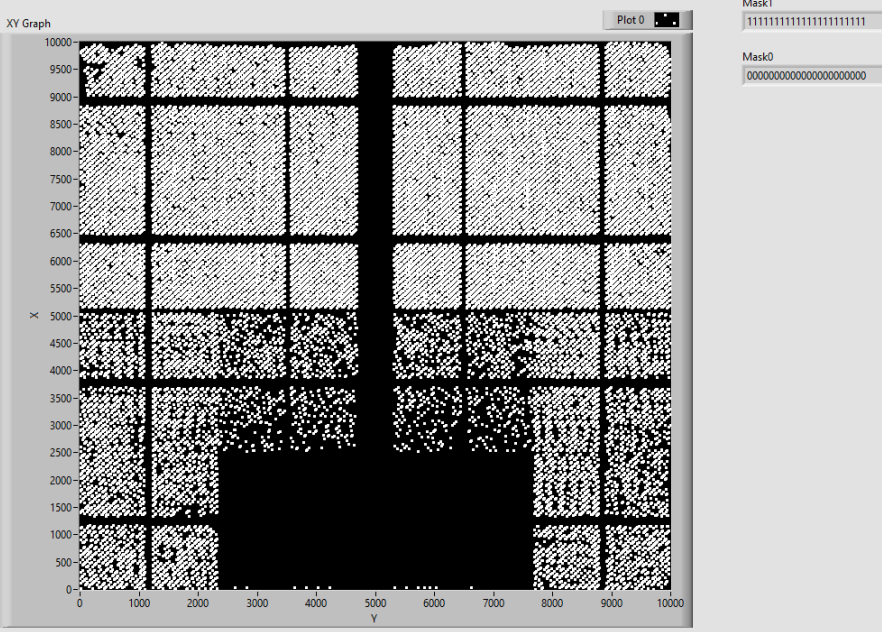

Mask0

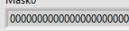

Рис. 5. График координат облученных ячеек памяти

(Fig. 5. The plot of the coordinates of the irradiated memory cells)

Анализ полученной карты проводился с помощью программы формирования карты сбоев и привёл к следующим выводам:

- При переборе значений трех старших разрядов адреса наблюдается разделение микросхемы на 8 пар равных блоков памяти симметричных относительно оси ординат (B0В7 на рис. 6).

- При переборе значений трех старших и трех младших разрядов наблюдается разделение микросхемы на 64 равных сегмента памяти размером $128 \times 512$ бит.

- При переборе значений разрядов А0-А6 наблюдается перемещение координат относительно оси ординат.

- При переборе значений разрядов А7-A15 наблюдается перемещение координат относительно оси абсцисс.

Схематическое изображение полученных закономерностей представлено на рис. 6.

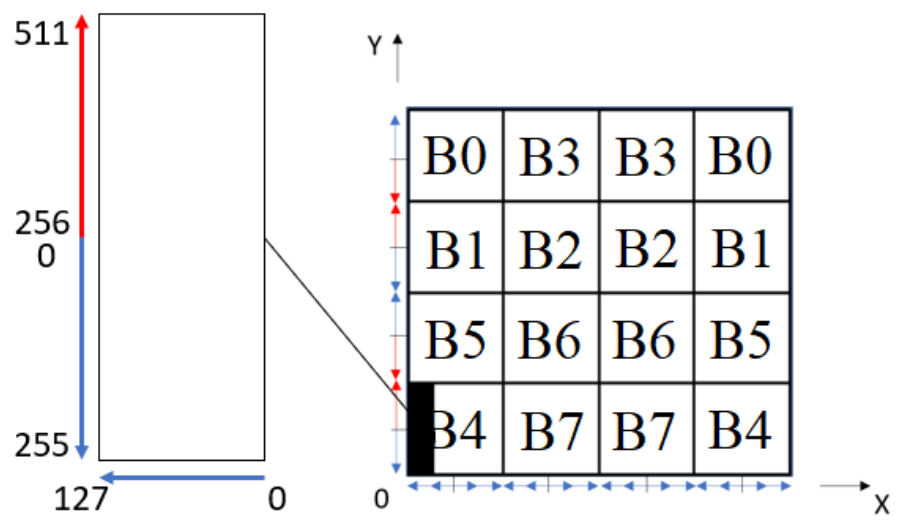

Рис. 6. Схематическое изображение полученных закономерностей расположения ячеек памяти

(Fig. 6. Schematic representation of the obtained patterns of the locations of memory cells) 
Вячеслав А. Чепов, Иван И. Швецов-Шиловский, Сергей Б. Шмаков

Стрелки вдоль осей абсцисс и ординат показывают, в каком направлении происходит смещение координат при увеличении значения адреса. Таким образом, учитывая симметрию расположения блоков и сегментов памяти, можно однозначно устанавливать расположение ячеек памяти с точностью до 1-го бита информации.

Результаты, полученные в ходе анализа, при сравнении с данными от изготовителя подтвердились.

\section{6. Применение алгоритма для анализа результатов при воздействии импульсного ИИ}

Воздействие проводилось на источнике лазерного излучения РАДОН-8, предназначенного для моделирования эффектов мощности дозы ионизирующего излучения на интегральные схемы. В ходе эксперимента контролировалась сохранность информации микросхемы памяти. Воздействие проводилось на весь кристалл со стороны приборного слоя.

Результат, полученный при помощи программы вывода изображения в ходе эксперимента, представлен на рис. 7 (ячейки памяти, подвергшиеся воздействию импульсного ИИ выделены красным цветом, остальные ячейки - зеленым). Видно, что при одинаковом уровне воздействия на всю площадь кристалла, подавляющее большинство сбившихся ячеек памяти приходится на его центр. Отсюда вывод, что ячейки памяти, находящиеся ближе к центру, сбиваются при меньшем уровне воздействия чем те, которые находятся на периферии. Данный результат может быть полезен при исследованиях эффекта «просадки питания на внутренних шинах» (Rail span collapse) [4, 5].

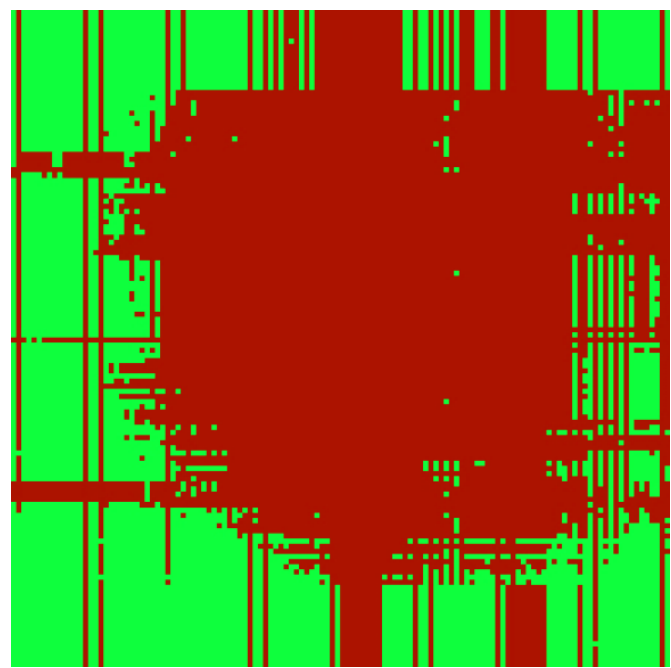

Рис. 7. Результат воздействия импульсного ИИ на микросхему СОЗУ (Fig. 7. The result of the impact of pulsed ionizing radiation on the SRAM chip)

\section{Заключение}

В статье продемонстрирован разработанный алгоритм соотнесения физической и логической адресаций в микросхемах памяти с помощью источников лазерного излучения, а также представлен аппаратно-программный комплекс для его реализации. Разработан инструмент визуализации карты сбоев для возможности оперативного анализа на карте логической и физической адресаций.

Проведена апробация алгоритма на сфокусированной лазерной установке при заданных диаметре пятна 3 мкм и шаге сканирования 100 мкм в микросхеме СОЗУ 


\section{Вячеслав А. Чепов, Иван И. Швецов-Шиловский, Сергей Б. Шмаков АЛГОРИТМ СООТНЕСЕНИЯ ФИЗИЧЕСКОЙ И ЛОГИЧЕСКОЙ АДРЕСАЦИЙ В МИКРОСХЕМАХ ПАМЯТИ С ПОМОЩЬЮ ИСТОЧНИКОВ ЛАЗЕРНОГО ИЗЛУЧЕНИЯ}

ёмкостью 4 Мбит с проектными нормами 180 нм. Результаты апробации выведены на карту сбоев.

Проведён анализ особенностей топологии исследуемого объекта при помощи данных на карте сбоев, полученных на сфокусированной лазерной установке. Результаты анализа подтвердили возможность разработки системы с точностью до 1 бита информации.

Представлено применение инструмента визуализации карты сбоев на примере исследования стойкости микросхемы СОЗУ к воздействию импульсного ИИ на источнике лазерного излучения РАДОН-8.

\section{СПИСОК ЛИТЕРАТУРЫ:}

1. Чумаков, А.И. Радиационная стойкость изделий ЭКБ [Текст] / А.И. Чумаков // Общая характеристика ионизирующих излучений: сб. статей. - М.: НИЯУ МИФИ, 2015. - 512 с.

2. Барбашов, В.М.; Трушкин, Н.С. Оценка надежности цифровых ис при воздействии радиации. Безопасность информационных технологий, [S.1.]. Т. 23, № 3. С. 11-19, oct. 2016. ISSN 2074-7136. URL: https://bit.mephi.ru/index.php/bit/article/view/13 (дата обращения: 15.08.2020).

3. Романова И.К. Современные методы визуализации многомерных данных: анализ, классификация, реализация, приложения в технических системах [Текст] / И.К. Романов - М.:, Наука и Образование. МГТУ им. Н.Э. Баумана. Электрон. журн. 2016. № 03. С. 133-167. URL: $\quad$ https://cyberleninka.ru/article/n/sovremennye-metody-vizualizatsii-mnogomernyh-dannyh-analizklassifikatsiya-realizatsiya-prilozheniya-v-tehnicheskih-sistemah (дата обращения: 15.08.2020).

4. A.B. Boruzdina et al., "Temperature Dependence of MCU Sensitivity in $65 \mathrm{~nm}$ CMOS SRAM," in IEEE Transactions on Nuclear Science. Vol. 62, no. 6. P. 2860-2866, Dec. 2015. DOI: https://doi.org/10.1109/TNS.2015.2499120.

5. D.G. Mavis, D.R. Alexander and G.L. Dinger, "A chip-level modeling approach for rail span collapse and survivability analyses," in IEEE Transactions on Nuclear Science. Vol. 36, no. 6. P. 2239-2246, Dec. 1989. DOI: https://doi.org/10.1109/23.45430.

6. Согоян А.В., Чумаков А.И. Диффузионная модель ионизационной реакции элементов БИС при воздействии ТЗЧ, «Микроэлектроника». // Микроэлектроника. 2017. Т. 46. № 4. С. 305-312. DOI: https://doi.org/10.7868/S0544126917040081.

7. P.K. Skorobogatov, G.G. Davydov, A.A. Pechenkin, D.V. Boychenko. Behavior of modern integrated circuits after latch-up parrying. RAD Conference Proceedings. Vol. 2. P. 159-162, 2017. DOI: https://doi.org/10.21175/RadProc.2017.32.

8. Боруздина А.Б. Методики экспериментальных исследований многократных сбоев в КМОП микросхемах статических оперативных запоминающих устройств при возведении отдельных ядерных частиц [Текст]: автореферат диссертации на соиск. учен. степ. канд. техн. наук (05.13.05) / Боруздина Анна Борисовна; НИЯУ «МИФИ». - Москва, 2015. - 25 с.

9. J.N. Bradford, "Geometric Analysis of Soft Errors and Oxide Damage Produced by Heavy Cosmic Rays and Alpha Particles," in IEEE Transactions on Nuclear Science. Vol. 27, no. 1. P. 941-947, Feb. 1980. DOI: https://doi.org/10.1109/TNS.1980.4330955.

10. D. Giot, P. Roche, G. Gasiot, J. Autran and R. Harboe-Sorensen, "Heavy Ion Testing and 3-D Simulations of Multiple Cell Upset in $65 \mathrm{~nm}$ Standard SRAMs," in IEEE Transactions on Nuclear Science. Vol. 55, no. 4. P. 2048-2054, Aug. 2008. DOI: https://doi.org/10.1109/TNS.2008.916063.

11. Чумаков, Александр И. Возможности и ограничения лазерных методов при оценке параметров чувствительности бис к эффектам воздействия тяжелых заряженных частиц. Безопасность информационных технологий, [S.1.]. Т. 26, № 3. С. 58-67, сен. 2019. ISSN 2074-7136. URL: $\quad$ https://bit.mephi.ru/index.php/bit/article/view/1217 (дата обращения: 15.08.2020). DOI: http://dx.doi.org/10.26583/bit.2019.3.05.

12. Лазерная установка ПИКО-3. URL: http://www.spels.ru/index.php?option=com_content\&view=article\&id $=344: 2017-04-20-09-07-42 \&$ catid=43:ntk\&Itemid=54. (дата обращения: 15.08 .2020 ).

13. Лазерная установка РАДОН-8. URL: http://www.spels.ru/index.php?option=com_content\&view=article\&id $=346: 2017-04-20-09-07-42 \& c a t i d=43:$ ntk\&Itemid=54 (обращения: 15.08 .2020 ).

\section{REFERENCES:}

[1] Chumakov A.I. Radiation resistance of electronic component base products. General characteristics of ionizing radiation: collection of articles., Moscow: National research nuclear University MEPhI, 2015. - 512 p. (in Russian). 


\section{Вячеслав А. Чепов, Иван И. Швецов-Шиловский, Сергей Б. Шмаков АЛГОРИТМ СООТНЕСЕНИЯ ФИЗИЧЕСКОЙ И ЛОГИЧЕСКОЙ АДРЕСАЦИЙ В МИКРОСХЕМАХ ПАМЯТИ С ПОМОЩЬЮ ИСТОЧНИКОВ ЛАЗЕРНОГО ИЗЛУЧЕНИЯ}

[2] Barbashov, V.M.; Trushkin, N.S. The digital ics reliability assessment under the influence of radiation. IT Security (Russia), [S.1.]. V. 23, no. 3. P. 11-19, oct. 2016. ISSN 2074-7136. URL: https://bit.mephi.ru/index.php/bit/article/view/13 (accessed: 15.08.2020) (in Russian).

[3] Romanova I.K. Modern Methods of Multidimensional Data Visualization: Analysis, Classification, Implementation, and Applications in Technical Systems. Science and Education of the Bauman MSTU, 2016, no. 03. P. 133-167. URL: https://cyberleninka.ru/article/n/sovremennye-metody-vizualizatsii-mnogomernyhdannyh-analiz-klassifikatsiya-realizatsiya-prilozheniya-v-tehnicheskih-sistemah (accessed: 15.08.2020) (in Russian).

[4] A.B. Boruzdina et al., "Temperature Dependence of MCU Sensitivity in $65 \mathrm{~nm}$ CMOS SRAM," in IEEE Transactions on Nuclear Science. Vol. 62, no. 6. P. 2860-2866, Dec. 2015. DOI: https://doi.org/10.1109/TNS.2015.2499120.

[5] D.G. Mavis, D.R. Alexander and G.L. Dinger, "A chip-level modeling approach for rail span collapse and survivability analyses," in IEEE Transactions on Nuclear Science. Vol. 36, no. 6. P. 2239-2246, Dec. 1989. DOI: https://doi.org/10.1109/23.45430.

[6] Sogoyan A.V., CHumakov A.I. Diffusion model of the ionization reaction of BIS elements under the influence of TCH, "Microelectronics". Mikroelektronika. 2017. Vol. 46, no 4. P. 305-312. DOI: https://doi.org/10.7868/S0544126917040081 (in Russian).

[7] P.K. Skorobogatov, G.G. Davydov, A.A. Pechenkin, D.V. Boychenko. Behavior of modern integrated circuits after latch-up parrying. RAD Conference Proceedings. Vol. 2. P.159-162, 2017. DOI: https://doi.org/10.21175/RadProc.2017.32.

[8] Boruzdina A.B. Methods of experimental research of multiple failures in CMOS chips of static RAM storage devices under the influence of individual nuclear particles: abstract of the dissertation for the competition. scientist. step. Cand. tech. sciences (05.13.05) / Boruzdina Anna Borisovna; Moscow: National research nuclear University MEPhI, 2015. $-25 \mathrm{p}$.

[9] J.N. Bradford, "Geometric Analysis of Soft Errors and Oxide Damage Produced by Heavy Cosmic Rays and Alpha Particles," in IEEE Transactions on Nuclear Science. Vol. 27, no. 1. P. 941-947, Feb. 1980. DOI: https://doi.org/10.1109/TNS.1980.4330955.

[10] D. Giot, P. Roche, G. Gasiot, J. Autran and R. Harboe-Sorensen, "Heavy Ion Testing and 3-D Simulations of Multiple Cell Upset in $65 \mathrm{~nm}$ Standard SRAMs," in IEEE Transactions on Nuclear Science. Vol. 55, no. 4. P. 2048-2054, Aug. 2008. DOI: https://doi.org/10.1109/TNS.2008.916063.

[11] Chumakov, Alexander I. Possibilities and limitations of focused laser technique application for SEE sensitivity parameters estimation. IT Security (Russia), [S.1.]. V. 26, no 3. P. 58-67, sep. 2019. ISSN 2074-7136. URL: $\quad$ https://bit.mephi.ru/index.php/bit/article/view/1217 $\quad$ (accessed: 15.08.2020). DOI: http://dx.doi.org/10.26583/bit.2019.3.05.

[12] Laser installation PIKO-3. URL: http://www.spels.ru/index.php?option=com_content\&view=article\&id $=344: 2017-04-20-09-07-42 \&$ catid $=43:$ ntk\&Itemid=54. (date of access 15.08.2020) (in Russian).

[13] Laser installation RADON-8. URL: http://www.spels.ru/index.php?option=com_content\&view=article\&id $=346: 2017-04-20-09-07-42 \&$ catid $=43:$ ntk\&Itemid=54 (date of access 15.08.2020) (in Russian).

Поступила в редакцию - 26 июля 2020 г. Окончательный вариант - 20 августа 2020 г. Received - July 26, 2020. The final version-August 20, 2020. 